\title{
EFFECTS OF QUADRUPOLE ROTATIONS ON THE TRANSPORT OF SPACE-CHARGE-DOMINATED BEAMS: THEORY AND SIMULATIONS COMPARING LINACS WITH CIRCULAR MACHINES
}

\author{
R. A. Kishek, IPR, University of Maryland, College Park, MD, \\ J. J. Barnard and D. P. Grote, Lawrence Livermore National Lab, CA
}

\begin{abstract}
PIC-code simulation results are presented where a spacecharge-dominated beam is transported in a lattice with quadrupole rotation errors. Two examples are studied in detail: the circular lattice for the University of Maryland Electron Ring (UMER) and the straight lattice from an early design of a proposed heavy ion inertial fusion (HIF) research experiment known as the Integrated Research Experiment (IRE). Reasonably small errors have little effect. However, the dependence on the strength of the errors is nearly quadratic. Slightly larger errors, therefore, can cause deterioration in beam quality, as manifest by a large increase in beam emittance and formation of a halo, leading eventually to particle losses. The simulations are accompanied by the moment equations for such a system in the presence of beam acceleration.
\end{abstract}

\section{INTRODUCTION}

Many applications, from heavy ion inertial fusion to high energy colliders, are relying increasingly on high brightness, space-charge-dominated, beams. To maintain the brightness of the beam during transport, it is important to control lattice errors so as to minimize emittance growth. Accelerator lattice errors may give rise to envelope mismatches and mismatches provide a source of free energy which, if thermalized, can result in emittance growth and hence brightness degradation. Quadrupole rotation errors are an interesting class of accelerator element misalignments, because the two transverse ( $\mathrm{x}$ and $\mathrm{y}$ ) equations of motion become coupled at linear order in the coordinates.

We perform simulations using the WARP code [1] to investigate emittance growth under the presence of quadrupole rotation errors. Two generalized emittances (defined below) give a measure of the phase space occupied by the beam and so give a measure of inherent beam-quality that would be ultimately achievable after compensating skew (rotated) quadrupoles undo the cumulative effects of small random rotation errors of the focusing quadrupoles. The simulations address issues such as reversibility of emittance growth, dependence on space charge, role of nonlinearities, periodicity of errors, and acceleration. For this paper, we will use simulations performed on two machines: the University of Maryland Electron Ring (UMER) [2] and the Integrated Research Experiment in heavy ion fusion (IRE) [3].

\section{GENERALIZED EMITTANCE}

When quadrupole rotation errors are present, the $\mathrm{x}$ and $\mathrm{y}$ normalized emittances are not conserved, even for a beam with an initial Kapchinskij-Vladimirskij (K-V) distribution with a linear space charge force profile propagating under linear external forces. However, if the equations of motion result from linear forces and are derivable from a Hamiltonian system, constants of the motion may be obtained analogous to the normalized $\mathrm{x}$ and y emittances [4]. Further, the K-V distribution has been generalized [5] to distributions in which the principal axes do not align with the $\mathrm{x}$ and $\mathrm{y}$ axes, and moment equations have been derived [6] that assume the space-charge profile remains linear, consistent with the assumption of the KV-like distribution of ref. [5]. In ref. [6], a drifting, non-relativistic beam was assumed, and a conservation constraint was derived that is equivalent to the first of the conservation constraints in ref. [4].

In this section, we generalize slightly the moment equations of ref. [6] to include acceleration, and we evaluate both the first and second independent emittance-like conservation constraints of ref. [5]. In the presence of non-linearities, either from space-charge or the external focusing field, the underlying assumption of a linear force profile is violated, and therefore the derived constraints will evolve (usually increasing) along the accelerator. For simplicity we consider nonrelativistic beams. We assume the space charge force can be calculated from that of a beam with elliptical symmetry but that is rotated with respect to the $\mathrm{z}$ (longitudinal) axis. Using the same notation as ref. [6] the transverse ( $\mathrm{x}$ and $\mathrm{y}$ ) equations of motion are:

$$
\begin{aligned}
d^{2} x / d z^{2} & =K_{q x x} x+K_{q x y} y+K_{S x x}(x-\langle x>) \\
& +K_{S x y}\left(y-\langle y>)-\left(d \ln \beta_{z} / d z\right) x^{\prime}\right. \\
d^{2} y / d z^{2} & =K_{q y y} y+K_{q x y} x+K_{S y y}(y-\langle y>) \\
& +K_{S x y}(x-\langle x\rangle)-\left(d \ln \beta_{z} / d z\right) y^{\prime}
\end{aligned}
$$

Here $\beta_{z} \mathrm{c}$ is the longitudinal velocity, and $K$ with leading subscript $\mathrm{q}$ is associated with external focusing from quadrupoles whereas $K$ with leading subscript $\mathrm{s}$ result from space charge (cf. ref. 6.)

As in ref. [6], we may derive a set of ten first order equations for the quadratic moments of the distribution, here generalized slightly from ref. [6] to include acceleration (the operator $\Delta \mathrm{ab} \equiv\langle\mathrm{ab}\rangle-\langle\mathrm{a}\rangle\langle\mathrm{b}\rangle$ ):

$d \Delta x^{2} / d z=2 \Delta x x^{\prime}$ 


$$
\begin{aligned}
d \Delta x x^{\prime} / d z= & \Delta x^{\prime 2}+K_{x x} \Delta x^{2}+K_{x y} \Delta x y-\left(d \ln \beta_{z} / d z\right) \Delta x x^{\prime} \\
d \Delta x^{\prime 2} / d z= & 2 K_{x x} \Delta x x^{\prime}+2 K_{x y} \Delta x^{\prime} y-2\left(d \ln \beta_{z} / d z\right) \Delta x^{\prime 2} \\
d \Delta y^{2} / d z= & 2 \Delta y y^{\prime} \\
d \Delta y y^{\prime} / d z= & \Delta y^{\prime 2}+K_{y y} \Delta y^{2}+K_{x y} \Delta x y-\left(d \ln \beta_{z} / d z\right) \Delta y y^{\prime} \\
d \Delta y^{\prime 2} / d z= & 2 K_{y y} \Delta y y^{\prime}+2 K_{x y} \Delta x y^{\prime}-2\left(d \ln \beta_{z} / d z\right) \Delta y^{\prime 2} \\
d \Delta x y / d z= & \Delta x y^{\prime}+\Delta x^{\prime} y \\
d \Delta x^{\prime} y / d z= & \Delta x^{\prime} y^{\prime}+K_{x x} \Delta x y+K_{x y} \Delta y^{2}-\left(d \ln \beta_{z} / d z\right) \Delta x^{\prime} y \\
d \Delta x y^{\prime} / d z= & \Delta x^{\prime} y^{\prime}+K_{y y} \Delta x y+K_{x y} \Delta x^{2}-\left(d \ln \beta_{z} / d z\right) \Delta x y^{\prime} \\
d \Delta x^{\prime} y^{\prime} / d z= & K_{x x} \Delta x y^{\prime}+K_{x y} \Delta y y^{\prime}+K_{y y} \Delta x^{\prime} y+K_{x y} \Delta x x^{\prime} \\
& \quad-2\left(d \ln \beta_{z} / d z\right) \Delta x^{\prime} y^{\prime}
\end{aligned}
$$

Here $K_{x x}=K_{q x x}+K_{s x x}, K_{x y}=K_{q x y}+K_{s x y}$, and $K_{y y}=K_{q y y}+K_{s y y}$. Using the procedures in ref. [4] we obtain the following invariants which we denote as

$$
\begin{aligned}
\varepsilon_{n g}^{2}= & \frac{1}{2}\left(\varepsilon_{n x}^{2}+\varepsilon_{n y}^{2}\right)+16 \beta^{2}\left(\Delta x y \Delta x^{\prime} y^{\prime}-\Delta x y^{\prime} \Delta x^{\prime} y\right) \\
\varepsilon_{n h}^{2}= & \left(\varepsilon_{n x}^{2} \varepsilon_{n y}^{2}+(4 \beta)^{4}\left(\left[\Delta x y \Delta x^{\prime} y^{\prime}\right]^{2}+\left[\Delta x y^{\prime} \Delta x^{\prime} y\right]^{2}\right.\right. \\
& -\Delta x^{2} \Delta y^{2}\left[\Delta x^{\prime} y^{\prime}\right]^{2}-\Delta x^{2} \Delta y^{\prime 2}\left[\Delta x^{\prime} y\right]^{2} \\
& -\Delta x^{\prime 2} \Delta y^{2}\left[\Delta x y^{\prime}\right]^{2}-\Delta x^{\prime 2} \Delta y^{\prime 2}[\Delta x y]^{2} \\
& -2 \Delta x y \Delta x y^{\prime} \Delta x^{\prime} y \Delta x^{\prime} y^{\prime}+2 \Delta x x^{\prime} \Delta y^{\prime 2} \Delta x y \Delta x^{\prime} y \\
& -2 \Delta x x^{\prime} \Delta y y^{\prime} \Delta x y \Delta x^{\prime} y^{\prime}-2 \Delta x x^{\prime} \Delta y y^{\prime} \Delta x y^{\prime} \Delta x^{\prime} y \\
& +2 \Delta x^{\prime 2} \Delta y y^{\prime} \Delta x y \Delta x y^{\prime}+2 \Delta x^{2} \Delta y y^{\prime} \Delta x^{\prime} y \Delta x^{\prime} y^{\prime} \\
& \left.\left.+2 \Delta x x^{\prime} \Delta y^{2} \Delta x^{\prime} y^{\prime} \Delta x y^{\prime}\right)\right)^{1 / 2}
\end{aligned}
$$

Here, $\quad \varepsilon_{n x}^{2} \equiv 16 \beta^{2}\left(\Delta x^{2} \Delta x^{\prime 2}-\left[\Delta x x^{\prime}\right]^{2}\right)$ and $\varepsilon_{n y}^{2} \equiv 16 \beta^{2}\left(\Delta y^{2} \Delta y^{\prime 2}-\left[\Delta y y^{\prime}\right]^{2}\right)$ are the squares of the usual $\mathrm{x}$ and $\mathrm{y}$ normalized emittances. Note that the square of the generalized emittances $\varepsilon_{n g}^{2}$ and $\varepsilon_{n h}^{2}$ reduce to the arithmetic and geometric mean of $\varepsilon_{n x}^{2}$ and $\varepsilon_{n y}^{2}$, respectively, in the absence of cross-correlations (i.e. $\Delta x y=\Delta x^{\prime} y=\Delta x y^{\prime}=\Delta x^{\prime} y^{\prime}=0$ ), and so form two independent quantities.

\section{SIMULATIONS}

Of particular interest is the response of actual machines, such as UMER and the IRE, to quadrupole rotation errors. Early injector experiments at the University of Maryland, for example, indicated a high sensitivity to quadrupole rotations. UMER is a circular machine consisting of 36 FODO cells and 36 bending dipoles. The nominal operating point of $10 \mathrm{keV}$ and $100 \mathrm{~mA}$ results in a generalized perveance of 0.0015 . With a normalized emittance, $\varepsilon_{\mathrm{nx}}$ of $10 \mathrm{~mm}$-mrad, this places the beam in the highly space-charge-dominated regime, with a tune-depression $\left(v / v_{0}\right)$ of 0.14 . The average beam size is about $1.0 \mathrm{~cm}$. For the moment, we have explored only the case of a drifting beam. The IRE on the other hand is an induction linac. A number of $\mathrm{K}+$ ion beams are injected at $1.6 \mathrm{MeV}$ into a strong-focusing lattice and then accelerated in parallel to $200 \mathrm{MeV}$. The generalized perveance at injection is similar to that of UMER
(0.0015). The quantity $\varepsilon_{\mathrm{nx}}$ is $1.0 \mathrm{~mm}$-mrad, placing it further than UMER in the space-charge-dominated regime. The average beam size is $1.5 \mathrm{~cm}$. Please see refs. [2-3] for further detail. For the simulations, we used the $2 \mathrm{~d} 3 \mathrm{v}$ version of WARP, with a resolution of 256 cells across the beam pipe ( 125 cells across the beam). In most cases we ran with 20,000 particles, and Gaussian filtering to reduce numerical collisions. We chose a semi-gaussian initial distribution in most cases so as to model a physical beam, although we occasionally used a $\mathrm{K}-\mathrm{V}$ distribution to compare against the theory.

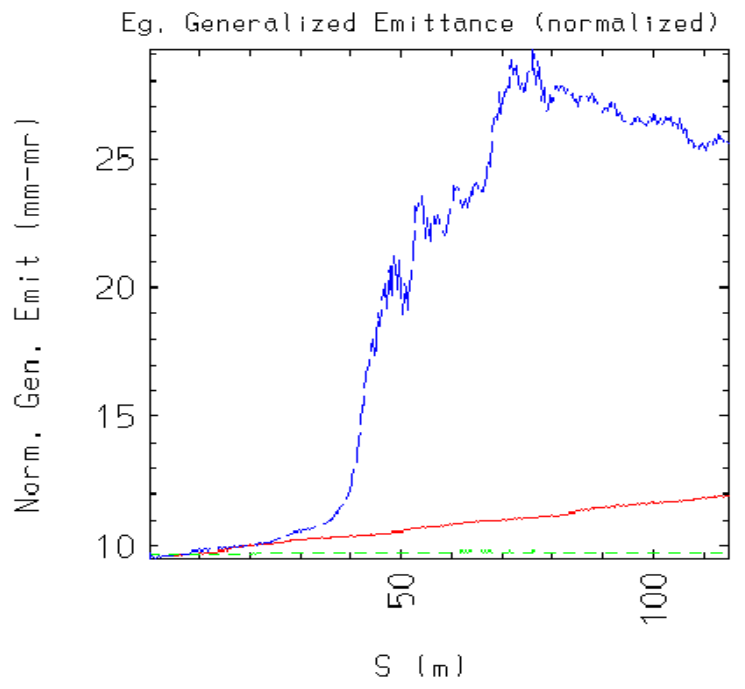

Figure 1: $\varepsilon_{\mathrm{ng}}$ along 10 turns in UMER; low current (lower); nominal current, straight (upper) and periodic (middle curve).

The simplest cases simulated are those of a drifting beam in a straight lattice with linear magnets. To isolate the effect of space charge, we compared our simulation to an equivalent emittance-dominated beam obtained by reducing the current and increasing the emittance to maintain the same beam size, while keeping the external forces unchanged. To both cases, we applied the same random distribution of errors, with an rms width of $0.2^{\circ}$ ( $\sim 4 \mathrm{mrad})$. As shown in the bottom curve in Fig. 1, the low-current beam exhibited almost no growth in the generalized emittance. The $\mathrm{x}$ and $\mathrm{y}$ rms emittances oscillated about their initial values. The beam rotation angle performs a random walk yet remains constrained within $3^{\circ}(\mathrm{rms})$ from upright. Increasing the space charge to the nominal parameters of UMER results in markedly different behavior. The beam responds to the same set of errors by "wobbling" more violently, with the beam rotation angle exceeding $5^{\circ}$ and continuing to increase in amplitude of oscillation. These large rotation angles translate into rms mismatches in $\mathrm{x}$ and $\mathrm{y}$, with associated growth of the generalized [top curve of Fig. 1]. The rate of growth is slow initially, but experiences a sudden boost as a halo forms, then levels off as the beamhalo system reaches a new equilibrium. 
A drifting beam in a ring (as in UMER) experiences random errors that repeat periodically every turn. This periodicity could introduce resonances that will further degrade the beam. Imposing this periodicity on our UMER simulation, however, resulted in remarkably different behavior. The periodicity of the errors imposes a periodicity on the beam rotation angle, preventing it from growing uncontrollably. Thus the rms mismatch in our case does not grow to a sufficient level to induce a halo. Therefore, the generalized emittance [middle curve, Fig. 1] grows steadily, but does not experience the abrupt growth during the $4^{\text {th }}$ turn. Note that we have only explored one set of parameters. It may be possible to see different behavior if we operate near a resonance.

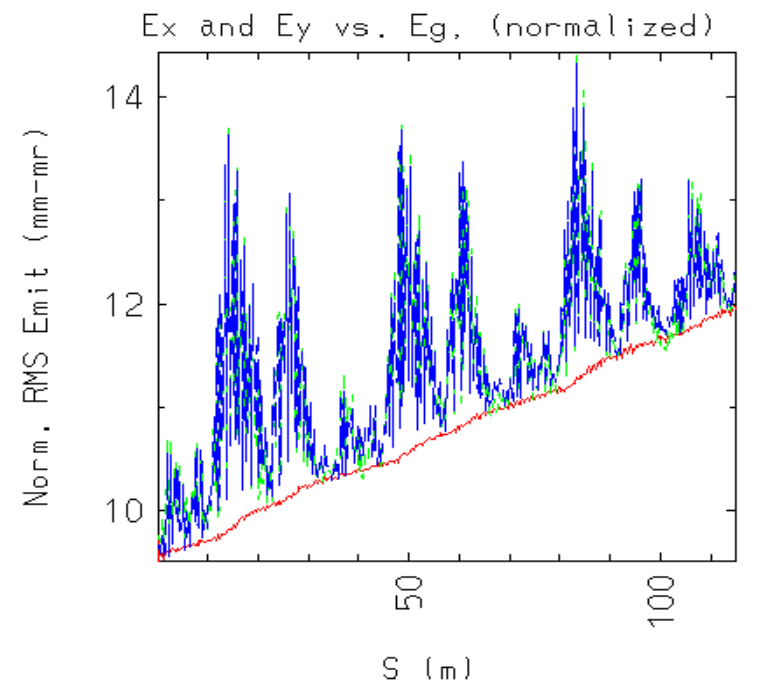

Figure 2: $\varepsilon_{\mathrm{nx}}, \varepsilon_{\mathrm{ny}}$ (dotted) and $\varepsilon_{\mathrm{ng}}$ (solid) for 10 turns of UMER.

The emittance shown in Fig. 1 is the generalized emittance, $\varepsilon_{\mathrm{ng}}$. The standard $\varepsilon_{\mathrm{nx}}$ and $\varepsilon_{\mathrm{ny}}$ follow a different behavior. Figure 2 compares $\varepsilon_{\mathrm{nx}}$ with $\varepsilon_{\mathrm{ng}}$ for the case of periodic errors shown in Fig. 1. Whereas the generalized emittance grows secularly and gradually, the standard $x$ emittance oscillates wildly. The oscillations are due to the fact that the beam is wobbling, and some of the apparent emittance growth is reversible. Note that $\varepsilon_{\mathrm{ng}}$ acts as a lower bound for $\varepsilon_{\mathrm{nx}}$, thus representing the nonreversible part of the emittance growth.

The beam rotation angle, as well as the emittance growth, depends sensitively on the magnitude of the errors. Figure 3 displays the evolution of $\varepsilon_{\mathrm{nh}}$ in the IRE for 2 values of errors. In both cases, an abrupt transition occurs at the point where halo formation takes place. In the case with larger errors, the halo formation is more severe (i.e., a larger fraction of the particles form the halo). It is evident that for smaller errors and prior to the halo formation, the generalized emittance is more nearly constant. The case with no errors is included to mark the numerical growth. By improving the numerics, we can obtain near zero emittance growth for the error-free case. Note that the dependence on the numerics can change in the presence of errors, as preliminary evidence seems to indicate. Hence we are pursuing the matter further.

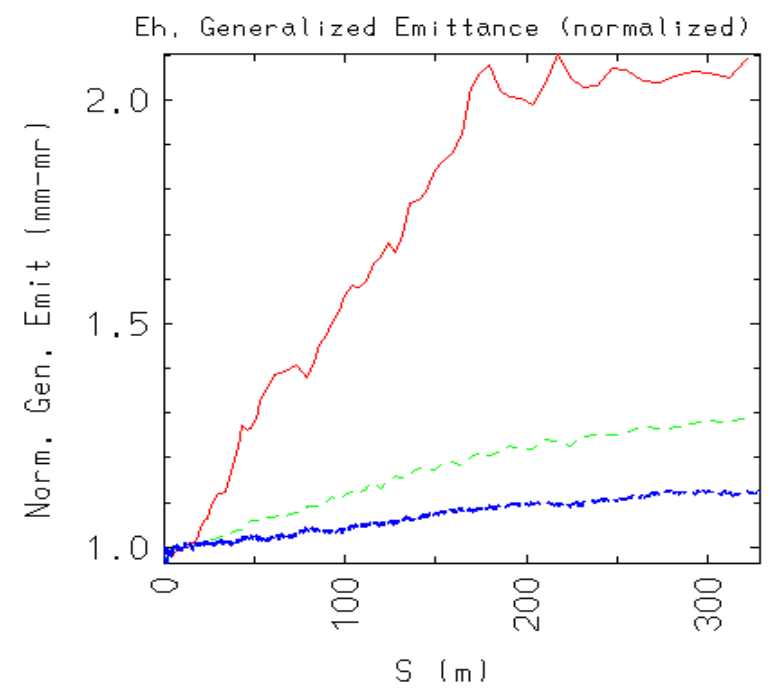

Figure 3: $\varepsilon_{\text {nh }}$ along the IRE for an rms error of $0.2^{\circ}$ (upper), $0.1^{\circ}$ (middle) and no error (lower curve).

\section{CONCLUSIONS}

As we find from the simulations presented here, quadrupoles with small random rotations have a larger impact on higher intensity beams. We presented an analytic derivation of a generalized emittance, which is conserved in linear systems. With the introduction of nonlinearities in the space charge distribution as the beam evolves, these generalized emittances are found to grow, sometimes dramatically if a halo is formed. An interesting effect is observed if the errors are periodic, as in a beam drifting in a ring, where the beam's response to the errors appears to be periodic and bounded.

\section{ACKNOWLEDGEMENTS}

We wish to acknowledge the assistance of both the Maryland Electron Ring Team, especially M. Reiser, P. O'Shea, and S. Bernal, as well as the IRE simulations working group, especially A. Friedman and R. Bangerter. Thanks especially to I. Haber who belongs to both groups. Thanks also to K. Danylevich for assistance.

Research Supported by Department of Energy under contracts DE-FG02-92ER54178, DE-FG02-94ER40855, and W-7405-ENG-48.

\section{REFERENCES}

[1] D. P. Grote, et. al., Fus. Eng. \& Des. 32-33, 193-200 (1996).

[2] M. Reiser, et. al., these proceedings, TUCR3; also R. A. Kishek, et. al., these proceedings, TUP118.

[3] A. Friedman, et. al., these proceedings, TUP144.

[4] Dragt, A.J., Neri, F., and Rangarajan, G., Phys. Rev. A, 45, 2572, (1992.)

[5] Sacherer, F.J., Ph.D. Thesis, Univ. of California, Berkeley, UCRL18454 (1968).

[6] Barnard, John J., Proc. 1995 PAC Conf. (Dallas, TX) 5, p. 3241, (1996) 